\title{
Anatomical variations of the Ulnar Artery
}

\author{
Abeywardhana $\mathrm{UNY}^{1}$, Wahalawatte $\mathrm{YR}^{1}$, Dissanayake $\mathrm{JK}^{1}$, Amaratunga $\mathrm{HA}^{1}$ \\ ${ }^{\prime}$ Department of Anatomy, Faculty of Medicine, University of Peradeniya. \\ *niroabey@yahoo.com
}

The arterial distribution of the upper

limb shows a large number of

anatomical variations $(9 \%-18.5 \%)$.

Knowledge about these variations is important during surgeries, intraarterial injections, venipuncture and imaging of the upper limb. With this background a study was conducted on 54 upper limb specimens of 27 cadavers during routine dissection at the Department of Anatomy, Faculty of Medicine, Peradeniya. The anterior compartment of the arm, cubital fossa and the forearm were dissected accordingly, and the pathway and the distribution of the ulnar artery were documented. Variations of the ulnar artery anatomy were noted in 2 specimens (3.7\%). Unilateral superficial ulnar artery was noted in one of them. The ulnar artery commenced at the level of the neck of the radius and passed superficial to the pronator teres, fibrous arch of the flexor digitorum superficialis, flexor carpi ulnaris and continued lateral to flexor carpi ulnaris tendon at the distal forearm. In the other specimen, the brachial artery trifurcated into the ulnar, radial and common interosseous arteries at the level of the neck of the radius. The ulnar artery continued superficial to the flexor digitorum superficialis, flexor carpi ulnaris up to mid forearm ( $7 \mathrm{~cm}$ from trifurcation) and then ran deep into the plane between flexor carpi ulnaris and flexor digitorum profundus. The superficial course of the ulnar artery was noted in two specimens. It increases the risk of arterial injury especially during venipuncture. Reporting of such variations in different populations is important for the practicing clinicians during diagnostic and surgical procedures. 